\title{
CYBERNETIC BASIS AND SYSTEM PRACTICE OF REMOTE SENSING AND SPATIAL INFORMATION SCIENCE
}

\author{
Xiang TAN ${ }^{\text {a,b,c }}$, Xin JING ${ }^{\text {d }}$, Rui CHEN ${ }^{\text {a,b }}$, Zhaohui MING ${ }^{\text {c }}$, Liqin HE ${ }^{\text {a,b }}$, Yiyuan SUN ${ }^{\text {a,b }}$, Xianfang SUN ${ }^{\text {e }}$, Lei YAN ${ }^{\text {a,b* }}$ \\ ${ }^{a}$ Institute of Remote Sensing and GIS, School of Earth and Space Science, Peking University, Beijing, 100871, China - \\ lyan@pku.edu.cn; (obbie1,sunyiyuan94)@163.com; heliqin_de@126.com \\ b Beijing Key Lab of Spatial Information Integration \& Its Applications, Peking University, Beijing 100871, China \\ ${ }^{c}$ Specialized Forces College of PAP, Beijing 102202, China - 598051000@qq.com \\ d South Dakota State University, South Dakota 57007, USA - vickyxin@126.com \\ e School of Computer Science \& Informatics, Cardiff University, United Kingdom - xianfang.sun@ cs.cardiff.ac.uk \\ *Corresponding Author: Lei YAN, E-mail: lyan@pku.edu.cn
}

Commission IV, WG IV/4

KEY WORDS: Remote Sensing, Spatial Information, Cybernetics, System Practice, Closed-loop control

\begin{abstract}
:
Cybernetics provides a new set of ideas and methods for the study of modern science, and it has been fully applied in many areas. However, few people have introduced cybernetics into the field of remote sensing. The paper is based on the imaging process of remote sensing system, introducing cybernetics into the field of remote sensing, establishing a space-time closed-loop control theory for the actual operation of remote sensing. The paper made the process of spatial information coherently, and improved the comprehensive efficiency of the space information from acquisition, procession, transformation to application. We not only describes the application of cybernetics in remote sensing platform control, sensor control, data processing control, but also in whole system of remote sensing imaging process control. We achieve the information of output back to the input to control the efficient operation of the entire system. This breakthrough combination of cybernetics science and remote sensing science will improve remote sensing science to a higher level.
\end{abstract}

\section{INTRODUCTION}

Since the American mathematician Norbert Wiener published 《cybernetics - on the science of communication and control of animals and machines》, cybernetics has been proposed for more than sixty years. Cybernetics provides a new set of ideas and methods for the study of modern science and promotes the transformation of contemporary philosophical ideas. Scholars in various fields have studied the cybernetics in their own fields constantly. Since then, cybernetics has developed various branches: biological cybernetics(and medical cybernetics), engineering cybernetics(and robotics cybernetics), social and economic cybernetics(and management cybernetics), military cybernetics, natural cybernetics(ecology, environment, resources, etc.) and others. Cybernetics is widely used in the fields of biology, medicine, physiology, psychology, ecology, environment, resources, economy, politics, military and social sciences and physics and chemistry in the natural sciences, which can lead to surprising new discoveries (Wu B W, 2008).

Remote sensing applications focus on the timeliness, emergency rescue also requires all-weather remote sensing monitoring, real-time has increasingly become the requirement of remote sensing technology applications. Real-time is essentially automatic. Real-time acquisition, processing and application of remote sensing information are all based on automatic control. The quantitative researches of remote sensing information have an important role in the development of remote sensing, and have become the frontier technology of remote sensing. It has many growth points and can promote the development of many disciplines and applications. Quantitative researches of remote sensing information involve the design and manufacture of remote sensing instruments, atmospheric detection and atmospheric parameter inversion, atmospheric impact correction methods and techniques, geo-station and geometric calibration methods and techniques, computer image processing, the setting of ground radiation and geometric calibration experiment fields and a variety of remote sensing application models and methods, geo-parameter inversion and projection and other disciplines and fields. Each link is very important, which has its own theory, methods and techniques. Only by making full use of the theory of systematic cybernetics and establishing the scientific relationship between input and output of each part can we make these areas coordinate and develop so as to finally solve the quantitative and real-time task of remote sensing information.

However, the quantification automatic control and analysis techniques of remote sensing time and spatial information remains a challenge. Although the target recognition and classification, intelligent human-computer interactive methods have been widely applied, the automation control and analysis and recognition mode, artificial intelligence, expert systems and other technologies are still not smart enough, the accuracy still needs to be improved. Therefore, the cybernetics introduction of the field of remote sensing, we need to start from the remote sensing system, and establish time-space closed-loop control theory of remote sensing actual operation. Then we should extend the theory of cybernetics to the specific examples, to achieve the coherent process of spatial information from the acquisition to the application. Finally it can provide a possibility for the significant increase of the overall effectiveness in spatial information acquisition, processing, transmission and application. 


\section{SYSTEMATIC CYBERNETICS THEORY IN REMOTE SENSING INFORMATION}

As a result of the development of remote sensing technology, along with the improvement of time and spatial resolution of remote sensing information, cybernetics is being applied into more fields. Automatic methods are becoming more and more popular, because the fully automatic can promote the real-time and on-orbit synchronization. So as to construct the sensor grid, and directly obtain useful data and information from the data back and on-orbit processing. And then to carry out automatic searches based on image content and change detection of specific target for achieving full automation as soon as possible.

To achieve all quantitative inversion of remote sensing is the main development trend with the comprehensive geometric and physical equations. As we all know, community needs puts forward a difficult challenge to the remote sensing workers, but also brings unprecedented development opportunities. It requires years of theoretical, technical and engineering accumulation, based on a variety of individual technical maturity. And there is a pattern formed with unified theoretical support, comprehensive technical means and advanced space integration information system. The most fundamental remote sensing information control problem can be solved.

\subsection{Basic Definition of Cybernetics Theory and Expression of Transfer Function Flow Diagram}

In cybernetics, "control" is defined as: in order to "improve" the function of one or some of the controlled objects. With the information obtained, the effects on the object can be confirmed. It shows that the control is based on the information. The information is transmitted in order to control, so any control depends on the feedback is to be achieved. Feedback is an extremely important concept in the theory of cybernetics. Frankly, information feedback refers to the control system sending out the information, but also receiving the information returned back. Information feedback also contains affecting the output and playing a regulatory role in achieving the intended purpose. Cybernetics is a scientific theory with methodological significance. The theory and view of cybernetics can be a scientific method for studying scientific problems. To imagine a discipline as a control system, by analysing the information flow, the feedback mechanism and control principles, the best state of the system can be found. So it just being called cybernetic thinking. The main methods of cybernetics are information methods, feedback methods, functional simulation methods, black box methods and white box methods.

The structure diagram manifests the form of the control system. It is composed of a number of boxes with a certain function relationship, according to the link between the various links in the system. The box will be linked together, and indicates the direction of the signal transmission. In the structure diagram, one end of the box is the input signal of the corresponding link, the other end is the output signal, the signal transmission direction is indicated by the arrow, and the function relation in the box is the transfer function of the corresponding link (Zhang $\mathrm{Q}, 2012)$. The structure diagram can express the composition of the system, the function of each link and the flow of the signal. Usually it's composed of four basic units, and just called the four elements of the structure diagram, respectively (Dong W Y et al., 2011):
1. Signal line: The signal line is a straight line with an arrow. Where the arrow indicates the flow of the signal, the time function of the signal or the phase function (ie, the Laplace transform) mark is on the straight line, as shown in Fig. 1 (a). The signal line marks the system variable.

2. Extraction point: The extraction point is also known as the branch point or measurement point, it is two or more signal outputs, indicating the signal leads or measured the location, as shown in Figure 1 (b). The same position leads to the same signal size and nature.

3. Comparison point: The comparison point is also referred to as the integration point or the addition point. It's two or more of the signal addition and subtraction (comparison) operation. "Ten" means additive, "one" means subtraction, "ten" can be omitted from writing, as shown in Figure 1 (c). Note: The amount of addition must have the same physical dimension.

4. Box: The box represents the function of the component or link between the input and the output function, as shown in Figure 1 (d). To write a symbol or a link in the box.

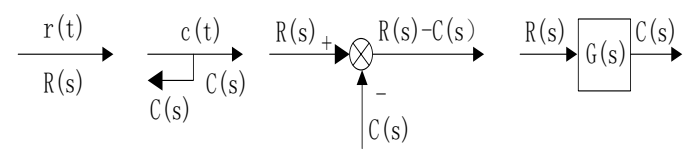

Figure 1. Structure diagram of control system four elements

\subsection{Spatial State Equation and Differential Form of Linear System}

\subsubsection{Space state equation}

The spatial state expression is based on the basic concept of state, state variables, state space, state equation and so on. State is a set of variables, in the case of known future input. It's possible to describe the set of the smallest number of all the movements in the system. For the general map plane studied by geographic information, it requires two separate states should be described. For the spatial information of concern for remote sensing or photogrammetry, $\mathrm{n}$ independent states are required to describe n-dimensional information. The state is the "position" in space, which is the basic coordinate that describes the movement of the system. An $\mathrm{n}$ order system, if it determines its motion state, should have at least $\mathrm{n}$ independent variables, that is, $\mathrm{n}$ state variables, with $\mathrm{x}_{1}(\mathrm{t}), \mathrm{x}_{2}(\mathrm{t}), \mathrm{x}_{3}(\mathrm{t}) \ldots, \mathrm{x}_{\mathrm{n}}(\mathrm{t})$ to represent $(\mathrm{T})=\left[\mathrm{x}_{1}(\mathrm{t}), \mathrm{x}_{2}(\mathrm{t}), \mathrm{x}_{3}(\mathrm{t}) \ldots, \mathrm{x}_{\mathrm{n}}(\mathrm{t})\right] \mathrm{T}$, the set of all state vectors, the set of state variables. The $\mathrm{n}$-dimensional space formed by the coordinates of each element in the state variable is geometrically the state space. That is, the state space is formed by all the state vectors $\mathrm{x}(\mathrm{t})$.

In the study, a state of the system can be regarded as a point in the state space. The initial state of the system is the initial point in the state space. The first order vector differential or difference equation describing the relationship between the system state variable and the input variable is called the state equation of the system and does not contain the input calculus term:

$$
\bar{x}=f(x, u, t)
$$


The output equation of the transformation relationship between the system variable group $\mathrm{x}$ and the input variable group $\mathrm{u}$ and the output variable group y is:

$$
y=g(x, u, t)
$$

The differential equation or transfer function describing for the input and output relations can be obtained experimentally. We can establish the state space description from the input and output relation, which is a way to establish the state space description.

\subsubsection{Linear system}

Through the mathematical model to study the automatic control system, we can get rid of the different characteristics of different types of systems for studying its inherent common movement law. Therefore, the establishment of remote sensing and geographic information science system of mathematical model, is the analysis and design of the control system of the primary work. Commonly used mathematical models are differential equations, transfer function, structure diagram, signal flow diagram, frequency characteristics and state space description. The mathematical model has a differential equation corresponding to the time domain, the transfer function corresponding to the complex field, and the frequency characteristic corresponding to the frequency domain.

The state equation of the linear system is the first order vector linear differential or difference equation, and the output equation is the vector algebraic equation. A linear system is a linear system whose properties do not change over time. It's a special case of a constant system, but as long as the nonlinearity of the system in the range of the study has little effect on the process of system motion, then the stationary system can be regarded as a linear stationary system. For a linear steadystate system, the response to the system output response is always the same, regardless of the time at which the input is added, as long as the input state variable is the same.

\subsubsection{Differential Equation of Control System}

The so-called mathematical model is a mathematical expression that indicates the dynamic relationship between physical quantities within the system. Reveal the internal structure of the system, parameters and performance characteristics of the intrinsic link. For example, towards a differential equation, if there is the same state variables, you can get the same output no matter which time you input the value. The remote sensing system can be analysed accordingly.

\subsection{Differential Equation's Laplace Algebraic Equation Transformation}

Laplace transform is an integral transformation commonly used in engineering mathematics. It is a simplified function of the establishment of the real variable function and complex variable function between a function transformation. For a real variable function for Laplace transform. Then the operation results for Laplace inverse transformation to obtain the real field in the corresponding results. It's much easier to calculate than the direct result in the real field.

The operation step of the Laplace transform is particularly effective for solving linear differential equations. It can be easy to solve the algebraic equation to deal with differential equation.
The calculation is simplified. In the classical control theory, the analysis and synthesis of the control system are based on the Laplace transform. It provides the possibility to use an intuitive and simple graphical approach to determine the overall characteristics of the control system, to analyse the motion of the control system, and to calibrate the integrated control system.

\section{THE APPLICATION OF CONTROL THEORY IN REMOTE SENSING OBSERVATION SYSTEM}

With the situation of the current remote sensing observation system is still not perfect, the researches on the real-time, intelligent ground observation system is very urgent at home and abroad. The section mainly describes remote sensing and control platform system; real-time intelligent model; UAV remote sensing equipment, automatic control system.

\subsection{Remote Sensing Platform}

Remote sensing platform refers to the loading of remote sensing sensors. Remote sensing platforms have many types according to the height from the ground to the platform. It mainly can be divided into three categories: ground platform, aviation platform and space platform. Remote sensing data with different area, different resolution, different characteristics and different uses can be obtained on different remote sensing platforms. In remote sensing applications, different height of the remote sensing platforms can be used alone or used in conjunction with the composition of three-dimensional remote sensing network. Common remote sensing platform in Table 1.

\begin{tabular}{|c|c|c|c|}
\hline Platform & Height & Purpose and use & Other \\
\hline $\begin{array}{l}\text { Static } \\
\text { satellite }\end{array}$ & $36000 \mathrm{~km}$ & $\begin{array}{l}\text { Fixed-point Earth } \\
\text { observation }\end{array}$ & $\begin{array}{l}\text { Weather } \\
\text { satellite }\end{array}$ \\
\hline $\begin{array}{c}\text { Earth } \\
\text { observation } \\
\text { satellite }\end{array}$ & $\begin{array}{c}500- \\
1000 \mathrm{~km}\end{array}$ & $\begin{array}{l}\text { Periodic earth } \\
\text { observation }\end{array}$ & $\begin{array}{l}\text { Landsat, } \\
\text { SPOT, etc. }\end{array}$ \\
\hline $\begin{array}{c}\text { Space } \\
\text { experiment } \\
\text { spacecraft }\end{array}$ & $\begin{array}{c}240- \\
350 \mathrm{~km}\end{array}$ & From time to time & \\
\hline $\begin{array}{l}\text { Return } \\
\text { satellite }\end{array}$ & $\begin{array}{c}200- \\
250 \mathrm{~km}\end{array}$ & $\begin{array}{l}\text { Reconnaissance } \\
\text { and } \\
\text { photogrammetry }\end{array}$ & \\
\hline $\begin{array}{l}\text { Wireless } \\
\text { radiosonde }\end{array}$ & $\begin{array}{l}100 \mathrm{~m}- \\
100 \mathrm{~km}\end{array}$ & $\begin{array}{l}\text { Various surveys } \\
\text { (weather, etc. ) }\end{array}$ & \\
\hline $\begin{array}{l}\text { High altitude } \\
\text { jet machine }\end{array}$ & $10-12 \mathrm{~km}$ & $\begin{array}{c}\text { Reconnaissance } \\
\text { with large range of } \\
\text { investigation }\end{array}$ & \\
\hline $\begin{array}{l}\text { Medium and } \\
\text { low altitude } \\
\text { aircraft }\end{array}$ & $\begin{array}{c}500- \\
8000 \mathrm{~m}\end{array}$ & $\begin{array}{c}\text { Aerial } \\
\text { photogrammetry, } \\
\text { various surveys }\end{array}$ & \\
\hline Airship & $\begin{array}{c}500- \\
3000 \mathrm{~m}\end{array}$ & $\begin{array}{l}\text { Air reconnaissance, } \\
\text { all kinds of } \\
\text { investigation }\end{array}$ & \\
\hline Helicopter & $\begin{array}{c}100- \\
2000 \mathrm{~m}\end{array}$ & $\begin{array}{l}\text { photogrammetry, } \\
\text { various surveys }\end{array}$ & \\
\hline
\end{tabular}




\begin{tabular}{|c|c|c|c|}
\hline Platform & Height & Purpose and use & Other \\
\hline $\begin{array}{l}\text { Wireless } \\
\text { remote } \\
\text { control } \\
\text { aircraft }\end{array}$ & $500 \mathrm{~m}$ & $\begin{array}{c}\text { Following } \\
\text { photogrammetry, } \\
\text { various } \\
\text { investigation }\end{array}$ & $\begin{array}{l}\text { Aircraft, } \\
\text { helicopter }\end{array}$ \\
\hline $\begin{array}{l}\text { Traction } \\
\text { aircraft }\end{array}$ & $50-500 \mathrm{~m}$ & $\begin{array}{l}\text { Photogrammetry, } \\
\text { various survey }\end{array}$ & $\begin{array}{l}\text { Traction } \\
\text { glider }\end{array}$ \\
\hline $\begin{array}{l}\text { Tethered } \\
\text { balloon }\end{array}$ & $800 \mathrm{~m}$ & $\begin{array}{c}\text { Following various } \\
\text { surveys }\end{array}$ & \\
\hline Ropeway & $10-40 \mathrm{~m}$ & site investigation & \\
\hline Crane & $5-50 \mathrm{~m}$ & $\begin{array}{c}\text { Close range } \\
\text { photogrammetry }\end{array}$ & \\
\hline $\begin{array}{l}\text { Ground } \\
\text { Survey Car }\end{array}$ & $0-30 \mathrm{~m}$ & Ground Condition & $\begin{array}{c}\text { Survey Car } \\
\text { Lifting } \\
\text { Platform }\end{array}$ \\
\hline
\end{tabular}

Table 1. Applicable remote sensing platform

\subsection{Real-time, Intelligent Model}

Integration and automation are the key to the real-time, intelligent model. It's the basis of intelligent means and method for real-time earth observation in China. It is the foundation of intelligent method, real-time earth observation, observation object and information processing.

Based on the existing working mode of ground observation system, space imaging detection and ground information processing are two key links. Multi-source data fusion, information intelligent, real-time processing and other aspects of the integration of links, in turn into the observation object, space observation network, and ultimately eliminate the artificial links, so as to establish the integration of time and space information real-time access to closed-loop model. So we can achieve observational control over airborne sensors. The main contents include:

1. Automatically serialized input process model: the mechanism of multi-source information co-processing in combination mode

Based on multi-source data imaging mechanism and signal correlation theory, scale space theory, algorithm adaptability and scheduling strategy, it is possible to achieve reliable and accurate registration of multi-source aerial imaging information. Then we can complete the registration of multi-source air and sky imaging information. At finally, to realize the collaborative data processing of multi-source imaging information with improving the efficiency, precision and automation of ground observation.

2. Automatic serialized output process model: imaging parameters non-stationary space-time stochastic model and joint solution mechanism

A experiment software system based on the optimal estimation of non-stationary space-time stochastic model and space-time imaging parameters can be established.

3. Observational object integration on automatic processing serialization model: spatial information spacetime validity evaluation mechanism

Based on the combination of the visual feature in the spatial domain and the transform domain, the combination of visual and non-visual features, the extraction of the category features, the visual feature perception model, the multi-scale hierarchical structure model, and so on. Analysis, quantification of temporal and spatial uncertainties of remote sensing information, and changes in temporal and spatial availability detection and typification.

4. Sensor with the automatic processing serialization model: aerospace observation network with flexible combination optimization principle and method

Considering the regular parameters of the platform in the ground observation network, the parameter system of the aerial observation network is optimized. Based on the above parameters system and the functional analysis and optimization theory, the flexible optimization combination of the aerial observation network is carried out.

5. Elimination of artificial links: sensor, observation object, automatic processing integration space-time closedloop control model simulation and major disaster observation target space-time sensor reconstruction feedback control simulation

With the theory of cybernetics, the feedback control of the system is realized by taking aerial observation data as input and taking evaluation of effectiveness as output. The system is analysed in time domain and frequency domain, static characteristic and dynamic characteristic analysis and system stability analysis; The earth observation time and space feedback control model simulation platform is established; The real-time and intelligent support for customization is provided.

Multi-source data fusion, information intelligent, real-time processing and other aspects of the integration of links, in turn into the observation object, space observation network, and ultimately eliminate the artificial links, so as to establish the integration of time and space information real-time access to closed-loop model. The various links were expressed in letters. It's expressed as follows: system input observation automation and major disaster demand $\mathrm{R}(\mathrm{s})$, to the final system output user/decision maker $\mathrm{C}(\mathrm{s})$. Through the sensor $\mathrm{Se}(\mathrm{s})$ and the sky observation platform $\mathrm{S}_{\mathrm{k}}(\mathrm{s})$, it forms the input of the sensor; From the sky to the ground, and it need to pass through the data link transmission $\mathrm{T}(\mathrm{s})$, the obtained information, multi-source information for collaborative processing $\operatorname{Pr}(\mathrm{s})$. The nonstationary imaging parameter $\mathrm{Pa}(\mathrm{s})$, after the observation of the object, the space-time validity evaluation of aerial imaging information $\mathrm{E}(\mathrm{s})$, the link has a negative feedback adjustment of the surface visual model $\mathrm{M}(\mathrm{s})$, the information fusion reconstruction $\mathrm{F}(\mathrm{s})$, the adjustment system, and finally the user/decision maker $\mathrm{C}(\mathrm{s})$. The letters are marked as shown above. The meaning of each parameter is: requirement $(\mathrm{R})$, Sensor (Se), space and sky observation platform $\left(\mathrm{Sky}, \mathrm{S}_{\mathrm{k}}\right)$, data link transmission $(\mathrm{T})$, multi-source information processing $(\mathrm{Pr})$, non-stationary imaging parameters $(\mathrm{Pa})$, spatial evaluation of spatial imaging information (E), surface visual perception model (M), information fusion reconstruction (F), Consumer (C). Using the Mason formula to solve the problem as follow:

$$
\begin{gathered}
\frac{C(s)}{R(s)}=\frac{\sum_{k=1}^{2} P_{k} \Delta_{k}}{\Delta}=\frac{P_{1}+P_{2}}{1-\left(L_{1}+L_{2}\right)}= \\
\frac{\Delta}{\mathrm{S}_{\mathrm{e}}(\mathrm{s}) \mathrm{S}_{\mathrm{k}}(\mathrm{s}) \mathrm{T}(\mathrm{s}) \operatorname{Pr}(\mathrm{s}) \operatorname{Pa}(\mathrm{s}) \mathrm{E}(\mathrm{s})+\mathrm{S}_{\mathrm{e}}(\mathrm{s}) \mathrm{S}_{\mathrm{k}}(\mathrm{s}) \mathrm{T}(\mathrm{s}) \operatorname{Pr}(\mathrm{s}) \mathrm{E}(\mathrm{s})} \\
\frac{1+\mathrm{S}_{\mathrm{e}}(\mathrm{s}) \mathrm{S}_{\mathrm{k}}(\mathrm{s}) \mathrm{T}(\mathrm{s}) \operatorname{Pr}(\mathrm{s}) \operatorname{Pa}(\mathrm{s}) \mathrm{E}(\mathrm{s}) \mathrm{M}(\mathrm{s}) \mathrm{F}(\mathrm{s})+\mathrm{S}_{\mathrm{e}}(\mathrm{s}) \mathrm{S}_{\mathrm{k}}(\mathrm{s}) \mathrm{T}(\mathrm{s}) \operatorname{Pr}(\mathrm{s}) \mathrm{E}(\mathrm{s}) \mathrm{M}(\mathrm{s}) \mathrm{F}(\mathrm{s})}{\text { Figure 2. Transfer function }}
\end{gathered}
$$

\subsection{Design of Airborne Multi-camera Control System for UAV}

The system is used for UAV aerial remote sensing control. With automatic operation, no human operation during the operation. 
All parameters involved should be configured before the aircraft takes off. If there is no configuration, the system should set the default value. The user is a participant in the UAV Aeronautical Air Control System project. Users should be the entire air control system processes, the relevant hardware and software operation is very familiar with the relevant parameters to understand its practical significance. The main function is achieving the control of the four D200 cameras, so the camera can send the image to the camera management IPC to store. And then with generating a fast view, it can send the information to the air control IPC.

\subsection{Other Applications}

\subsubsection{Closed-loop Control System Based On Feature Information And Space-borne Payload}

Remote sensing of hollow space information from the acquisition to the application has four resident links: airborne load, ground objects, digital terrain information, analysis and decision making. From the satellite and other remote sensing platform, the need for the relevant equipment load design and improvement of the sensor to obtain the information needed to quantify the digital, so that can be used for analysis and decision-making data, these four links are interlocking, Interact with each other.

At present, remote sensing information real-time application, to achieve automatic assessment, analysis of the biggest problems, can be summarized as spatial information flow automatically transmitted three breakpoints and an open-loop problem. The three breakpoints are: 1) the effectiveness is poor, the sky and sky load indicators in the pursuit of high resolution and completeness while ignoring the effectiveness of information conversion; 2) lack of automation, the actual image of the multihand digital way to obtain digital ground Information; 3) quantitative weak, assessment, analysis depends mainly on visual interpretation and experience. An open loop refers to the command decision on the airborne load operation without feedback to the automatic restraint capability.

On the basis of the integration of digital space information and the digital space information, it is necessary to realize the customization of the sky and sky load, so as to realize the closed-loop control from the sky-sky load-information acquisition-information application-space load. To achieve closed-loop control, we must study the reverse process in the system.

We can use the idea of cybernetics to build a closed-loop control system based on the integration of space-time load and spatial information acquisition, transmission, analysis and processing until the final application (command decision). This paper introduces the theory and method of cybernetics, deeply studies the four links in remote sensing information, solves the three breakpoints and open loop problems of remote sensing information from acquisition and transmission to the establishment of automatic control model of remote sensing information. In order to better realize the quantitative and realtime information of remote sensing information.

\subsubsection{Application of Control Theory in Multi-source Remote Sensing Information Fusion}

One of the classic applications of cybernetics in remote sensing science is the use of multi-source aerial remote sensing data for various applications.

When a natural disaster occurs, we tend to use the panchromatic images and multispectral images to monitor and evaluate the affected area. When a fire or flood occurs, we tend to use panchromatic images and Lidar images for quick positioning. Select the type of image will select the type of sensor to obtain the corresponding character real representation of the image, select the remote sensing image type, you can through data processing and processing to achieve multi-source remote sensing data fusion, and ultimately use these data to monitor. And our feedback link is a different remote sensing application requirements, which will change our choice of data sources and sensors. Therefore, in order to make the remote sensing system more stable, the purpose of remote sensing system is achieved through feedback, which makes our remote sensing system stabilize even in the case of external disturbance.

The control system includes airborne loads, actual features and acquired digital terrain information. The customization of the sky load is taken as the input of the control system, and the auxiliary command decision information is used as the output of the control system. The purpose of the closed-loop control system is to provide feedback of the output information to the input.

This system is a remote control system for remote sensing decision making. It is a kind of closed-loop control in control theory. It is widely used in remote sensing geographic information science. Such a system will be returned to the input as a controlled input as a controlled output in a certain manner and a control relationship is imposed on the input. First of all, according to different remote sensing information applications to choose a different type of command decision. For such applications, remote sensing sensor loads such as space satellites, airplanes and even unmanned aerial vehicle systems are selected. Through the digital imaging of the actual features, access to different data images, that is, multi-source remote sensing data. And then through the computer digital processing to generate the type of visual identifiable features, where the classification of features can be monitored by remote sensing science classification or unsupervised classification to be achieved. To this end has been generated for a particular application of remote sensing information data. The last step, that is, the decision-making link of the whole system, according to the different needs of auxiliary command decision information and then for the original sensor selection, surface observation and remote sensing information resources to be replaced.

\section{CONCLUSION}

Closed-loop control system has feedback link. Through the feedback system, the closed-loop control system can improve the accuracy and shorten the response time. The method is suitable for the system which requires short response time and high stability. In summary, cybernetics can be used in remote sensing. Not only can be used to control remote sensing platforms, sensors, data processing control, cybernetics can also be used to control the remote sensing imaging process. Cybernetics also plays a role in multi-source data fusion. With 
the use of cybernetics in remote sensing, the entire remote sensing system can achieve more automatic and quantitative. However, the paper doesn't use the state equation to reflect the various quantitative relationship between the system input and output. So it's the focus of our future researches on the application of cybernetics in remote sensing.

\section{REFERENCES}

Dong W Y, He H Y, 2011. The foundation of control theory. Wuhan: Wuhan university press, pp. 6-7.

Lu S Q, Lei Yan, Bing Zhang, and etc., 2007, The integration of UAV remote sensing system and its flight test research. Science of Surveying and Mapping, vol.ED-32, pp.84-86.

Sun X J, Lei B, Cheng Z Y, and etc., 2012, The application of the data processing operation control workflow in the remote sensing. Computer Engineering, vol.ED-38, pp.28-30.

Wang X, Huang L, 2012, The design and implementation of camera control system based on the ARM in airborne remote sensing. Observation and Control Technology, vol.ED-31, pp.60-63.

Wiener Norbert, 1961, Cybernetics or control and communication in the animal and the machine. Massachusetts: MIT press.

Wu B W, 2008, Sixty years of cybernetics since founding. Control Theory and Applications, vol.ED-25, pp.597-602.

Zhang Q, 2012, The teaching discussion of using block diagram to get the transfer function. Mechanical and Electrical Product Development and Innovation, 25 (6):181-182.

Zhou X Y, Zhao Q, 2013, The control of the three-axis inertial and stabilization platform's double speed loop in airborne remote sensing. Journal of Chinese Inertial Technology, vol.ED-21, pp.439-445.

\section{APPENDIX}

We need to realize the integration research of spatial equipment and digital spatial information, so as to realize the closed-loop control from spatial loading, information acquisition, information application return to spatial loading (the anticlockwise direction in figure 3). In order to realize closed-loop control, we must study the reverse process of the system (the clockwise direction in figure 3 .

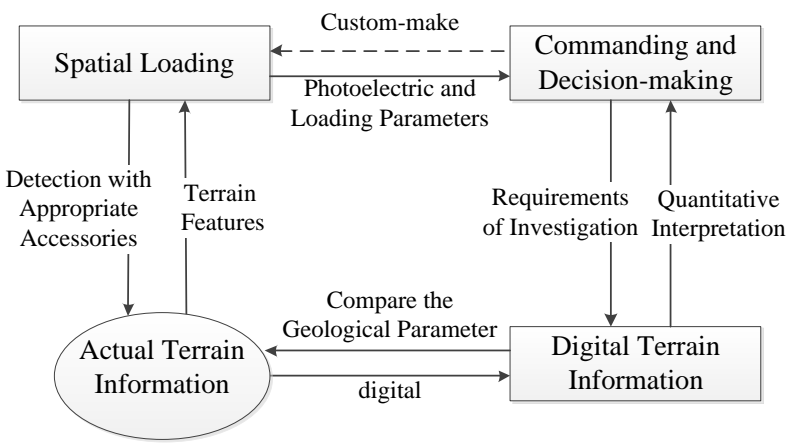

Figure 3. The closed-loop control system of spatial loading and feature information
We can build the integration research of spatial loading and information acquisition, procession, transformation, application base on the idea of cybernetics, and finally, construct a closedloop control system.

Figure 3 was showed as figure 4 in control flow diagram. This control system includes spatial loading, actual terrain information and digital terrain information. Regard custom of spatial loading as the input, and information of commanding and decision-making as the output. The purpose of closed-loop control system is to feed the output information back to the input.

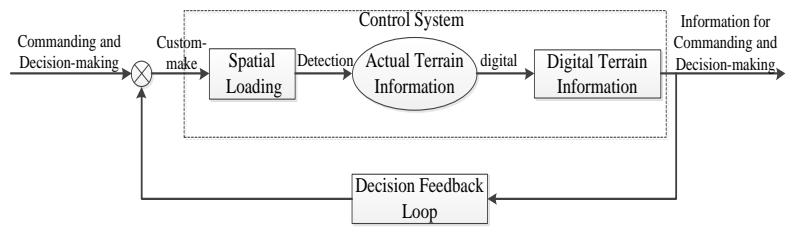

Figure 4. The closed-loop control system of spatial

The fusion of multi-source remote sensing data is another classic application of cybernetics in remote sensing science, as shown in figure 5.

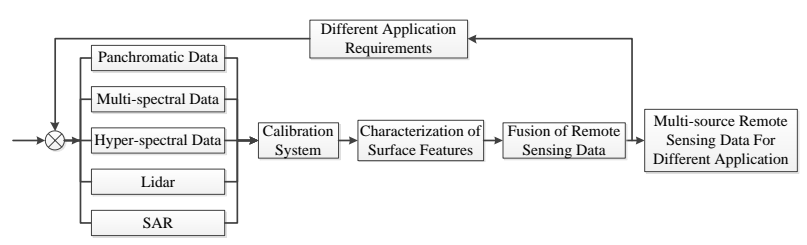

Figure 5. The application of cybernetics in multi-source remote sensing information fusion 